\title{
Combined laparoscopic-colonoscopic approach for the removal of a migrated intrauterine contraceptive device penetrating the sigmoid colon
}

Received: 9 February 2006 / Accepted: 30 April 2006 / Published online: 30 May 2006

(C) Springer-Verlag Berlin / Heidelberg 2006

\begin{abstract}
Uterine perforation is a rare but potentially lifethreatening complication associated with the use of intrauterine contraceptive devices (IUDs). Following perforation, the IUD can migrate to the peritoneal cavity or even perforate several adjacent organs. Migration to the sigmoid colon is extremely rare. We present the case of a 28-year-old woman who had an IUD inserted soon after delivery. The patient again became pregnant, but no IUD could be detected in routine examinations. After an asymptomatic interval of 3 years, the patient suffered acute abdominal pain. Initial laparoscopy elsewhere revealed the IUD attached to the sigmoid colon, but extraction was not possible. The patient was referred to us for further treatment. Under general anesthesia, laparoscopic exploration of the abdomen was performed. The IUD had perforated the sigmoid colon and was firmly fixed. In order not to injure the sigmoid colon, laparoscopic mobilization of the IUD was assisted with a "rendez-vous" sigmoidoscopy. The IUD was removed transanally with laparoscopic and sigmoidoscopic assistance, and the patient was discharged within $24 \mathrm{~h}$ after the operation. Insertion of an IUD necessitates regular checks to confirm the device's correct position. Migration of an IUD warrants prompt laparoscopic removal, even in asymptomatic patients.
\end{abstract}

Keywords Intrauterine contraceptive device - Migration · Sigmoid colon $\cdot$ Laparoscopy $\cdot$ Colonoscopy

J. Zieren $(\bowtie) \cdot$ B. Moebius $\cdot$ B. Zieren $\cdot$ C. Menenakos Department of General-, Visceral-, Vascular- and Thoracic Surgery, Charité, Campus Mitte, Humboldt University Berlin, Schumannstraße 20/21,

10117 Berlin, Germany

e-mail: juergen.zieren@charite.de

Tel.: +49-30-450522022

Fax: +49-30-450522905

\section{Introduction}

Copper $\mathrm{T}$ intrauterine devices (IUDs) have been used for more than 40 years as a contraceptive medium and remain the mainstay of family planning measures in developing countries. Use of these devices has been associated with serious complications, though. With an incidence of 1.2 per 1,000 insertions, uterine perforation is considered a rare complication [1]. Migration of an IUD can occur either into the peritoneal cavity or into adjacent organs such as the colon, with subsequent septic manifestations. Due of unspecific symptoms, many cases of migrated IUDs are diagnosed with delay or even accidentally. We report a case of IUD migration into the colonic wall. Following a laparoscopic dissection of intraabdominal adhesions, the device could be colonoscopically retrieved with a "rendezvous" maneuver.

\section{Case report}

A 28-year-old woman was fitted with a Multiload Cu375 IUD 3 months after an uncomplicated delivery. A routine check 1 month after implantation showed normal findings. Two months later the woman unexpectedly became pregnant again. Consecutive routine gynecological and ultrasound examinations during pregnancy failed to localize the implanted IUD in the uterine cavity. This was attributed to a spontaneous unnoticed expulsion of the IUD. The pregnancy and the second delivery were uncomplicated. Because the patient did not wish another pregnancy, oral contraceptive pills were initiated. Following an asymptomatic period of 3 years, the patient suddenly suffered an onset of acute pain in the left lumbar area mimicking kidney colic. Abdominal x-ray showed no nephrolithiasis, but surprisingly, the missed IUD was projected in the left lower abdomen (Fig. 1). Barium contrast studies were not done.

Biochemistry values were normal with no signs of inflammation. The patient was then submitted to a diagnostic laparoscopy by a gynecologist. Intraoperative 


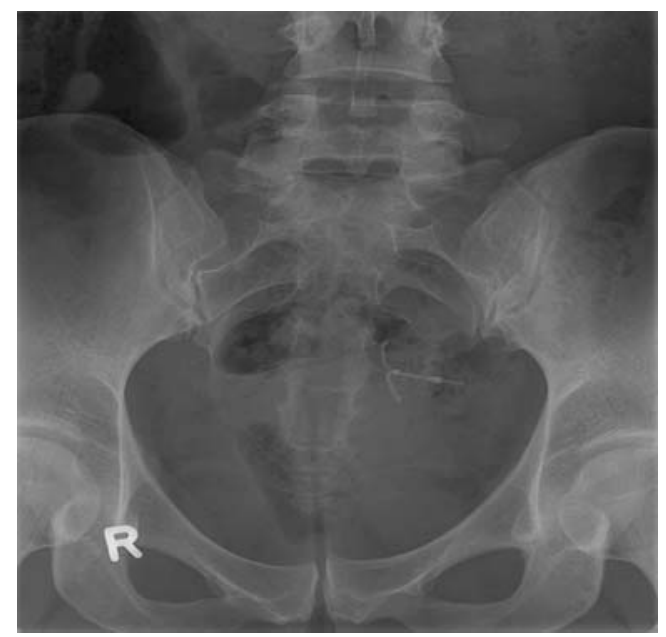

Fig. 1 Abdominal x-ray showing the migrated intrauterine device

findings were consistent with a complete migration of the IUD out of the uterus into the wall of the sigmoid colon. Neither abscess nor adhesions could be detected. Despite multiple attempts to detach the device from the colonic wall, the IUD could not be extracted. The patient was then referred to our department for further treatment.

Computed tomography of the abdomen showed no abscess. The patient was subsequently submitted to laparoscopic abdominal exploration, which confirmed the findings of the previous operation, namely a dislocated IUD partially fixed to the sigmoid wall (Fig. 2). After a careful dissection of the intraabdominal part of the device, we observed that one arm of the IUD had truly perforated the full thickness of the sigmoid colon, and the intraluminal part was firmly hooked in the colonic wall. Because removing the IUD posed specific difficulties, and to avoid a major enterotomy, we decided to perform a simultaneous "rendez-vous" sigmoidoscopy. It was also endoscopically confirmed that the intraluminal arm of the device was firmly attached in the sigmoid wall. With simultaneous laparoscopic and sigmoidoscopic manipulations, the IUD could be mobilized, and it was successfully removed transanally. The minimal perforation (about $1 \mathrm{~cm}$ ) of the sigmoid colon was laparoscopically sutured in two layers after meticulous lavage of the abdominal cavity. The sigmoidorectum was transanally insufflated, and there was no evidence of fluid or air leakage by laparoscopic visualization. The patient's postoperative course was uneventful, and she was discharged free of symptoms the next day.

\section{Discussion}

The cause of IUD migration is unclear. Besides primary false technique during the insertion of the device, a chronic inflammatory reaction with consecutive progressive erosion of the uterine wall seems to be important [2]. It usually occurs at the time of insertion of the device and is more common in the puerperium [1].

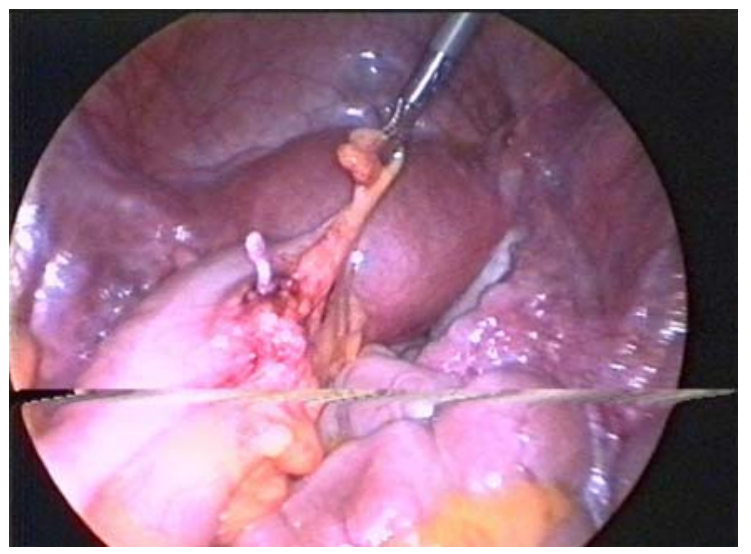

Fig. 2 Laparoscopic view of the migrated intrauterine device penetrating the sigmoid colon

Uterine perforation is a relatively rare complication, occurring in $1 / 350$ to $1 / 2,500$ insertions [3]. Perforation occurring at the time of insertion correlates directly with the skill of the clinician [3]. It can also occur if a previously inserted device is not removed in early pregnancy or is extruded at the time of delivery. The time of implantation soon after delivery seems to be a significant factor because the risk of uterine perforation seems to increase when the IUD is placed within 3 months after delivery $[4,5]$. It is commonly accepted that an IUD should be removed in pregnancy because of the increased risk of spontaneous abortion, septic complications, and premature delivery [1]. It is unknown why the device was inserted in our patient so soon after her delivery without a sufficient interval.

Perforation by IUD can also involve several adjacent organs such as the bladder [6] and, in particular, the intestinal tract, frequently resulting in serious septic complications [3]. The sigmoid colon represents the most frequent site of IUD migration in the intestinal tract $[1,7-$ 10], but other sites can be also affected, such as the appendix $[3,11]$ and the small intestine [12].

In our case, the patient reported no symptoms that could correlate with a possible device migration. Due to the limited number of cases reported in the literature, it is difficult to say whether there is a connection between the type or model of the IUD used and the risk of migration.

Laparotomy was previously the usual way to retrieve dislocated IUDs [13]. In the era of minimal invasive surgery, though, a migrated IUD can be successfully removed with laparoscopic and endoscopic techniques. Even when multiple adhesions prevent complete access to the dislocated device, laparoscopic dissection of these adhesions is, in most cases, achievable with eventual modification of the trocar sites. In the present case, laparoscopy was combined with a "rendez-vous" sigmoidoscopy. The IUD could then be completely mobilized and was transanally retrieved with minimal trauma to the sigmoid wall. Apparently, the device could have been removed laparoscopically without an additional endoscopy, but an enterotomy with subsequent complication risks and higher operating costs would then have been necessary. Colonoscopy could also confirm that the arm of the IUD 
had penetrated only one site on the sigmoid colon. Finally, transanal removal was thought to be prudent in order to avoid potential intraperitoneal contamination with laparoscopic retrieval.

In conclusion, the present case report emphasizes the need for regular sonographic checks of IUD implantation. The device may have migrated even if the patient is completely asymptomatic. A migrated device should be always removed, even in asymptomatic patients, because the risk of life-threatening complications is high [7]. The method of choice is laparoscopy, which could be combined with a colonoscopy if local conditions necessitate this bimodal approach. Minimal invasive techniques offer minimal tissue trauma and prompt rehabilitation after surgery.

\section{References}

1. Pirwany IR, Boddy K (1997) Colocolic fistula caused by a previously inserted intrauterine device. Case report. Contraception 56:337-339

2. Zakin D, Stern WZ, Rosenblatt R (1981) Complete and partial uterine perforation and embedding following insertion of intrauterine devices. I. Classification, complications, mechanism, incidence, and missing string. Obstet Gynecol Surv $36: 335-353$
3. Chang HM, Chen TW, Hsieh CB, Chen CJ, Yu JC, Liu YC, Shen KL, Chan DC (2005) Intrauterine contraceptive device appendicitis: a case report. World J Gastroenterol 11:5414 5415

4. Heartwell SF, Schlesselman S (1983) Risk of uterine perforation among users of intrauterine devices. Obstet Gynecol 61:31-36

5. Kiilholma P, Makinen J, Maenpaa J (199) Perforation of the uterus following IUD insertion in the puerperium. Adv Contracept 6:57-61

6. Zakin D (1984) Perforation of the bladder by the intrauterine device. Obstet Gynecol Surv 39:59-66

7. Tuncay YA, Tuncay E, Guzin K, Ozturk D, Omurcan C, Yucel $\mathrm{N}$ (2004) Transuterine migration as a complication of intrauterine contraceptive devices: six case reports. Eur J Contracept Reprod Health Care 9:194-200

8. Nceboz US, Ozcakir HT, Uyar Y, Caglar H (2003) Migration of an intrauterine contraceptive device to the sigmoid colon: a case report. Eur J Contracept Reprod Health Care 8:229-232

9. Abbasoglu O, Karakayali H, Akyurek M, Cebi Y (1994) Uterine perforation and ileosigmoid fistula caused by an intrauterine contraceptive device. Case report. Eur J Surg 160:189-190

10. Browning JJ, Bigrigg MA (1988) Recovery of the intrauterine contraceptive device from the sigmoid colon. Three case reports. Br J Obstet Gynaecol 95:530-532

11. Goldman JA, Peleg D, Feldberg D, Dicker D, Samuel N (1983) IUD appendicitis. Eur J Obstet Gynecol Reprod Biol 15:181183

12. Beard RJ (1981) Unusual presentation of translocated intrauterine contraceptive device. Lancet 1:837

13. Edelman DA, Berger GS (1980) Contraceptive practice and tuboovarian abscess. Am J Obstet Gynecol 138:541-544 